\title{
Surgical lung biopsy for the diagnosis of interstitial lung disease in England: 1997-2008
}

John P. Hutchinson, Tricia M. McKeever, Andrew W. Fogarty, Vidya Navaratnam and Richard B. Hubbard

Affiliation: Division of Epidemiology and Public Health, School of Medicine, University of Nottingham, Nottingham, UK.

Correspondence: John Hutchinson, C100 Clinical Sciences Building, University of Nottingham, City Hospital Campus, Hucknall Road, Nottingham NG5 1PB, UK. E-mail: john.hutchinsondanottingham.ac.uk

ABSTRACT International guidelines and new targeted therapies for idiopathic pulmonary fibrosis have increased the need for accurate diagnosis of interstitial lung disease (ILD), which may lead to more surgical lung biopsies. This study aimed to assess the risk of this procedure in patients from the UK.

We used Hospital Episodes Statistics data from 1997 to 2008 to assess the frequency of surgical lung biopsy for ILD in England, UK. We identified cardiothoracic surgical patients using International Classification of Diseases revision 10 codes for ILD and Office of Population Censuses and Surveys Classification of Interventions and Procedures version 4 codes for surgical lung biopsy. We excluded those with lung resections or lung cancer. We estimated in-hospital, 30-day and 90-day mortality following the procedure, and linked to cause of death using data from the UK Office of National Statistics.

We identified 2820 patients with ILD undergoing surgical lung biopsy during the 12-year period. The number of biopsies increased over the time period studied. In-hospital, 30-day and 90-day mortality were $1.7 \%, 2.4 \%$ and $3.9 \%$, respectively. Male sex, increasing age, increasing comorbidity and open surgery were risk factors for mortality.

Surgical lung biopsy for ILD has a similar mortality to lobectomy for lung cancer, and clinicians and patients should understand the likely risks involved.

@ERSpublications

30-day mortality after surgical lung biopsy for ILD is $\mathbf{2 . 4 \%}$ (similar to lobectomy for lung cancer) http://ow.ly/fQnY302fGvU

Editorial comment in Eur Respir J 2016; 48:1274-1277.

This article has supplementary material available from erj.ersjournals.com

Received: Feb 192016 | Accepted after revision: July 042016 | First published online: Sept 222016

Support statement: Funding for this work came from the University of Nottingham postgraduate support costs fund. R.B. Hubbard is the British Lung Foundation Professor of Respiratory Epidemiology and J.P. Hutchinson is funded by his Chair. Funding information for this article has been deposited with the Open Funder Registry.

Conflict of interest: Disclosures can be found alongside this article at erj.ersjournals.com

Copyright OERS 2016 


\section{Introduction}

Achieving an accurate diagnosis of interstitial lung disease (ILD) is important, as it can help guide treatment options and prognosis [1]. This is particularly true for idiopathic pulmonary fibrosis (IPF), where the introduction of pirfenidone and nintedanib as targeted therapies has the potential to slow the rate of decline in lung function, offering hope to patients who face a median survival of only 3 years $[2,3]$. While diagnosis can often be made after review of high-resolution computed tomography imaging at a multidisciplinary team meeting, a surgical lung biopsy may be required to confirm the histological diagnosis [1].

Surgical lung biopsy has associated risks, in part due to the impaired lung function of the patients involved. Case series have reported 30-day mortality rates of around $1.5-4.5 \%$, although these may be biased by careful case selection or local expertise and are not necessarily generalisable to other centres [4-7]. Other case series note much higher mortality rates $[8,9]$.

We have recently published data from a large secondary care dataset from the USA, identifying in-hospital mortality of $1.7 \%$ following elective surgical lung biopsy for ILD [10]. However, there are no comparable studies from Europe, and the USA study was not able to assess mortality following hospital discharge, meaning it is likely to underestimate the 30-day and 90-day mortality statistics commonly reported elsewhere. Here, we aim to assess the use of surgical lung biopsy for ILD in England, using a national secondary care dataset linked to national mortality statistics, to estimate the in-hospital, 30-day and 90-day mortality associated with the procedure.

\section{Methods}

We used the Hospital Episodes Statistics database, which contains details of all admissions to National Health Service (NHS) hospitals in England [11]. This is managed by the Health and Social Care Information Centre, a body linked to the UK Department of Health. We requested data on all admissions of patients with ILD (see online supplementary material for specific International Classification of Diseases codes used) from 1989 to 2008. We requested linked data from the Office of National Statistics (ONS) on date of death and underlying cause of death for all patients, where available, to establish mortality after discharge.

We excluded patients from years prior to 1997 as these did not have a unique identifier that allowed pairing with ONS data. We selected all patients who had undergone a surgical lung biopsy using the following Office of Population Censuses and Surveys Classification of Interventions and Procedures version 4 (OPCS-4) procedural codes: E593 (biopsy of lesion of lung), E552 (open excision of lung), E548 (other specified excision of lung) and E549 (unspecified excision of lung); in addition we required a treating specialty of "cardiothoracic surgery" to exclude patients undergoing radiological biopsies, who were unlikely to be under a surgical team. We identified additional codes suggestive of imaging involvement and excluded those mentioning "radiological", "ultrasound" or "computed tomography" approaches. We retained records specifying approach under "imaging" or "video" control, which could reflect video-assisted thoracoscopic surgery (VATS), but performed a sensitivity analysis excluding the less specific "imaging" codes. We identified codes for "open" and "thoracoscopic" procedures where available. We excluded any patients undergoing repeat operations, those with additional codes for lung resections that suggested the intention of the operation was therapeutic rather than diagnostic and also those with codes for lung cancer in the current or subsequent record, to ensure we did not include diagnostic procedures for the cancer rather than the ILD. We also excluded patients where surgical lung biopsy was not the primary operation coded and there was any doubt about the nature of the biopsy (see online supplementary material). Finally, we excluded any patient without a clear age or sex record.

We attempted to assess whether procedures were elective (scheduled) or nonelective (emergency). All patients with a decision-to-admit date prior to their actual admission date were classed as elective. Those without a valid decision-to-admit date or where this was the same as the operation date were classed as nonelective (see online supplementary material for further details). We explored the number of operations performed across England, as well as in-hospital, 30-day and 90-day mortality, cause of death, post-procedural complications, length of stay, and readmission rates. Complications were derived from additional diagnostic codes on the operation record that would be consistent with a post-operative complication; for conditions that could be a comorbidity (e.g. arrhythmia), these had to be absent from the preceding admission record (if available). We assessed the frequency of different types of operation (VATS or open thoracotomy) where this information was available and attempted to assess the impact of provisional type of ILD diagnosis, accepting this might be modified by subsequent biopsy results. Patients with more than one ILD diagnostic code were pragmatically coded as "unclassified” ILD.

We looked at risk factors for early death by logistic regression, adjusting for age, sex, level of comorbidity, level of deprivation, type of operation and provisional diagnosis. Comorbidities were derived from additional diagnostic codes present in either the operation record or previous records and scored using the 
updated Charlson score [12], a modified version of the Charlson Comorbidity Index [13] that takes into account advances in disease management since the original score was published almost 30 years ago. Using this score, patients with no or minor comorbidities are assigned a score of "0", whereas those with notable comorbidities receive points depending on the number and severity of comorbidities present. We categorised patients into those with scores of " 0 ", " 1 ", " 2 " and " $\geqslant 3$ ". Further information on how to calculate the updated Charlson score is available in online supplementary figure S1. Deprivation was measured using the Index of Multiple Deprivation 2010 (IMD) [14]. This score, which reflects indicators such as income, employment, education and crime (with a low score given to areas that are least deprived), was analysed as a continuous rather than categorical variable, as the latter was no more effective using the likelihood ratio test. Overall $\mathrm{p}$-values and $\mathrm{p}_{\text {trend-values were calculated using the likelihood ratio }}$ test. We assessed survival from the date of operation using the Cox proportional hazards model, with censoring of data in survivors on June 22, 2010 (last date of ONS data) or on date of lung transplantation, and examination of the proportional hazards assumption by the Schonfeld test. Statistical analysis was performed using Stata version 13.1 (StataCorp, College Station, TX, USA). Ethical approval for the use of the data was obtained from the NHS Health and Social Care Information Centre.

\section{Results}

After exclusions, our dataset contained 2820 patients with a diagnosis of ILD undergoing a surgical lung biopsy between 1997 and 2008 (figure 1). 55\% of these were male, with $73 \%$ aged $<65$ years. $81 \%$ of biopsies were classified as elective and $19 \%$ were nonelective. The number of biopsies increased over the years in the study period (table 1, and figures 2 and 3). The biopsy rate ranged from 0.27 to 0.74 per 100000 across the English regions (online supplementary table S1).

Codes specifying whether operations were performed via open thoracotomy or VATS were available for $38 \%$ of operations. Of these, $66 \%$ were VATS. No VATS codes were listed prior to 2006 , with $80 \%$ of patients having a code for the type of operation from 2007 onwards, suggesting the code for VATS came into practice at this time. The most common provisional diagnosis was J84.1 (the most specific code for IPF, but also including other idiopathic interstitial pneumonias), which comprised $50.8 \%$ of codes, followed by J84.9 ("unclassified ILD") with $28.8 \%$ of codes. This included 81 patients who had more than one diagnostic code listed.

Complications occurred in $13.9 \%$ of operations, based on discharge diagnoses for admissions with lung biopsies. The most common were pneumothorax (4.2\%), pneumonia (2.8\%), other unspecified complications of procedure (1.9\%), pleural effusion (1.4\%) and failed thoracoscopic approach with conversion to open surgery (1.2\%). $8.4 \%$ of patients with a valid record for critical care input spent time in a critical care area; for most this was a single stay, but 24 patients had more than one stay. The median (range) length of hospital stay was 4 (0-82) days. $14.1 \%$ of patients were readmitted to hospital within 3 months, with $28.0 \%$ of these having more than one readmission. Half of readmissions were due to ILD (table 2).

There were 911 deaths (32\% of the cohort) until the end of June 2010. The most common cause of death was ILD (50\%), followed by cancer (18\%) and cardiac disease (8\%) (table 3).

With regard to early deaths, in-hospital mortality was $1.7 \%$ (47 deaths), 30-day mortality was $2.4 \%$ (68 deaths) and 90-day mortality was 3.9\% (111 deaths). Elective biopsies had a lower mortality than nonelective biopsies: for elective procedures, in-hospital, 30 -day and 90 -day mortality were $1.0 \%, 1.5 \%$ and $2.8 \%$, respectively; for nonelective procedures, the figures were $4.6 \%, 6.3 \%$ and $8.8 \%$, respectively.

Risk factors for death within 90 days of biopsy (the time period yielding the most power) were identified as male sex, increasing age, increasing comorbidity and use of open thoracotomy (table 4). Results were broadly similar for deaths within 30 days or in-hospital, although the effect of comorbidity was stronger for in-hospital deaths (online supplementary tables S2 and S3). Risk factors were less significant when nonelective patients were excluded (online supplementary tables S4-S6). In a sensitivity analysis excluding 593 patients who had an additional procedure code specifying "approach to organ under imaging control" (which could be applied to VATS but was nonspecific and therefore unclear), mortality was slightly higher at $1.9 \%$ (in-hospital), $2.7 \%$ (30-day) and $4.2 \%$ (90-day). ILD was the most common cause of early death in each category. After splitting admissions into four time periods, mortality was lowest in the latest time period (2006-2008) for in-hospital, 30-day and 90-day mortality measures: in-hospital mortality of $1.0 \%$ in 2006-2008 versus $1.7 \%$ in 1997-1999, 30-day mortality of $1.8 \%$ in $2006-2008$ versus $3.0 \%$ in $1997-1999$ and 90 -day mortality of $2.8 \%$ in $2006-2008$ versus $4.3 \%$ in 1997-1999. Risk factors for death within 90 days of biopsy for the period 2005 onwards are presented in online supplementary table S7.

We calculated a rate of death of 6.81 (95\% CI 6.38-7.27) per 100 person-years in our biopsy cohort, suggesting that about $6 \%$ of patients would die in the first year after surgery. For those aged $\geqslant 65$ years, the rate of death was 13.14 (95\% CI 11.86-14.55) per 100 person-years, suggesting about $13 \%$ of patients would die in the first year. 


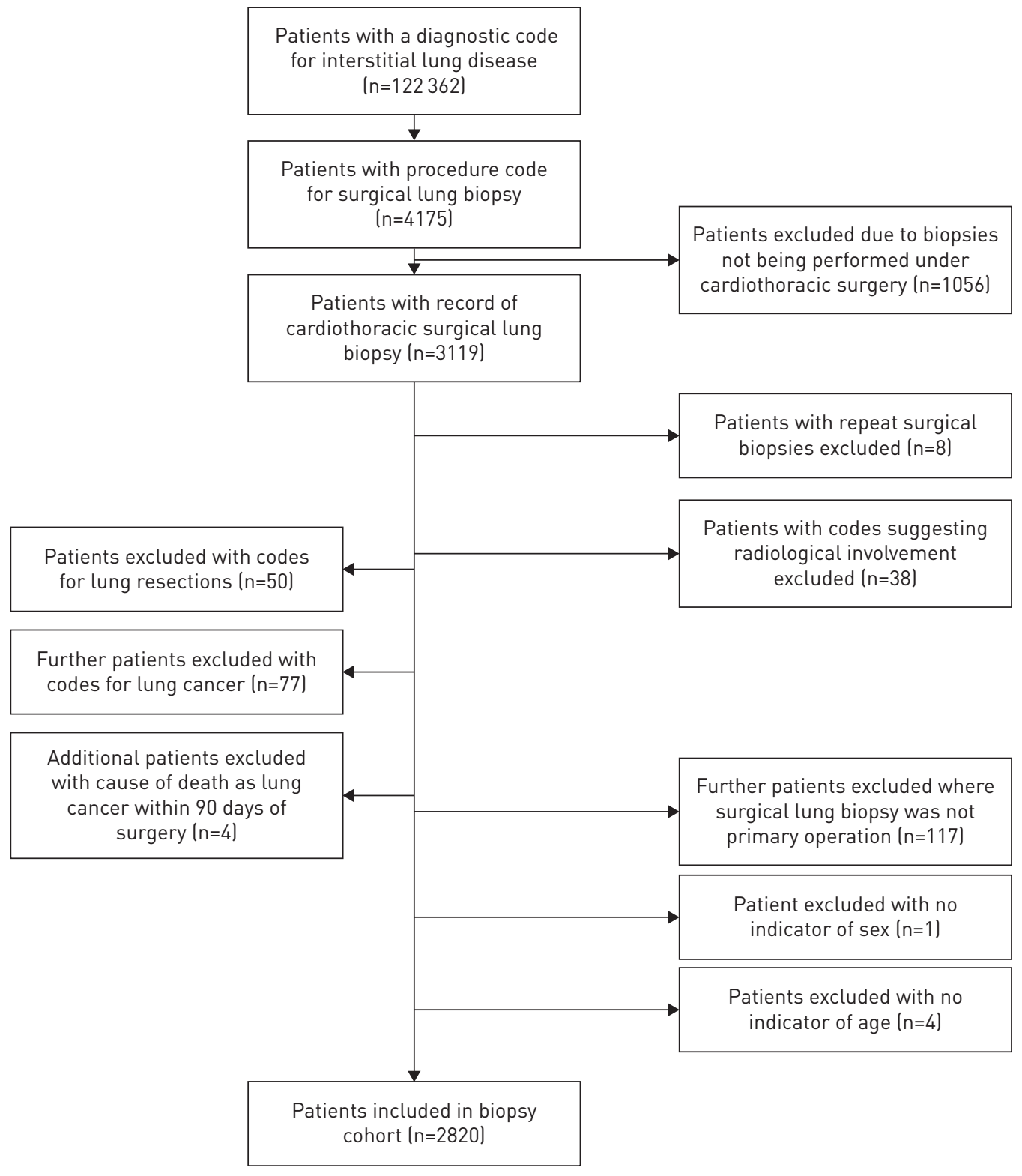

FIGURE 1 Flow diagram of patient selection.

Using Cox regression, we estimated that males had a 52\% increased risk of death compared to females, there was a 2 - to 7 -fold increased risk of death with increasing age compared to the lowest age category, there was an 2.3-fold increased risk of death with an updated Charlson score of $\geqslant 3$ compared with 0 and a $61 \%$ increased risk of death with open surgery compared with VATS (online supplementary table S8).

\section{Discussion}

Our data reveals an increasing number of surgical lung biopsies for ILD in England from 1997 to 2008, with variation according to geographical region. The figure of 367 biopsies in 2008 would equate to an average of 13 annual biopsies per thoracic surgical centre in England (see online supplementary material). Assuming our estimate of 51\% of biopsies being for a provisional diagnosis of J84.1 (most specific for IPF, but possibly including other idiopathic interstitial pneumonias), this would equate to around 187 biopsies per year for IPF-clinical syndrome, i.e. 4.5\% of the new cases per year in England (based on 5000 new cases in the UK [15], with England comprising 84\% of the UK population). Unsurprisingly, there was a higher mortality for nonelective admissions. Complications were reasonably common, and the most common cause for readmission and death was ILD (likely, but not certain, to represent acute exacerbations). 30-day mortality was $2.4 \%$, which is comparable to the 30 -day mortality following lobectomy for nonsmall cell lung cancer (2.3\%) (a potentially curative rather than diagnostic operation) [16]. Male sex, increasing age, increasing comorbidity and open surgery were risk factors for mortality. 


$\begin{array}{lr}\text { TABLE } 1 \text { Demographics of biopsy cohort } & \\ \text { Total patients } & 2820(100.0) \\ \text { Sex } & 1546(54.8) \\ \quad \text { Male } & 1274(45.2) \\ \text { Female } & \\ \text { Age group years } & 576(20.4) \\ \quad \leqslant 44 & 636(22.6) \\ 45-54 & 843(29.9) \\ 55-64 & 588(20.9) \\ 65-74 & 177(6.3) \\ >74 & \\ \text { Year of biopsy } & 104(3.7) \\ 1997 \text { (April onwards) } & 169(6.0) \\ 1998 & 191(6.8) \\ 1999 & 185(6.6) \\ 2000 & 213(7.6) \\ 2001 & 195(6.9) \\ 2002 & 197(7.0) \\ 2003 & 239(8.5) \\ 2004 & 262(9.3) \\ 2005 & 261(9.3) \\ 2006 & 345(12.2) \\ 2007 & 367(13.0) \\ 2008 & 92(3.3) \\ 2009 \text { (up until March) } & \end{array}$

Data are presented as $\mathrm{n}(\%)$.

Our cohort of 2820 patients is much larger than most case series of surgical lung biopsies for ILD and encompasses multiple centres from across a single country (England). By using the Hospital Episodes Statistics database, we have been able to comprehensively capture all records of admissions to NHS hospitals, representing the vast majority of patients who receive healthcare in England, and the ability to link this with national cause of death data means that we were able to reliably assess mortality after discharge. Therefore, unlike other series, we have been able to assess mortality at several stages, as well as readmissions, complications and ultimate cause of death for patients treated across a large number of surgical units.

However, there are limitations to our analysis. There is no clear diagnostic code for surgical lung biopsy in the OPCS-4 system and it is possible that some of our procedures were performed via another means, such as medical thoracoscopy or computed tomography-guided percutaneous needle biopsy. We attempted to exclude these by specifying cardiothoracic surgery as the treating speciality, but it is possible this may have been miscoded. The increasing number of cases in later years contrasts with a decrease in other studies [17] attributed to the publication of previous American Thoracic Society/European Respiratory Society consensus criteria [18]. It is possible we may have included some patients undergoing biopsy for malignancy, despite excluding all patients with codes for lung cancer; however, excluding all patients who subsequently died from cancer made little difference to our results. Our provisional diagnosis data should

FIGURE 2 Number of biopsies over time, stratified by sex.

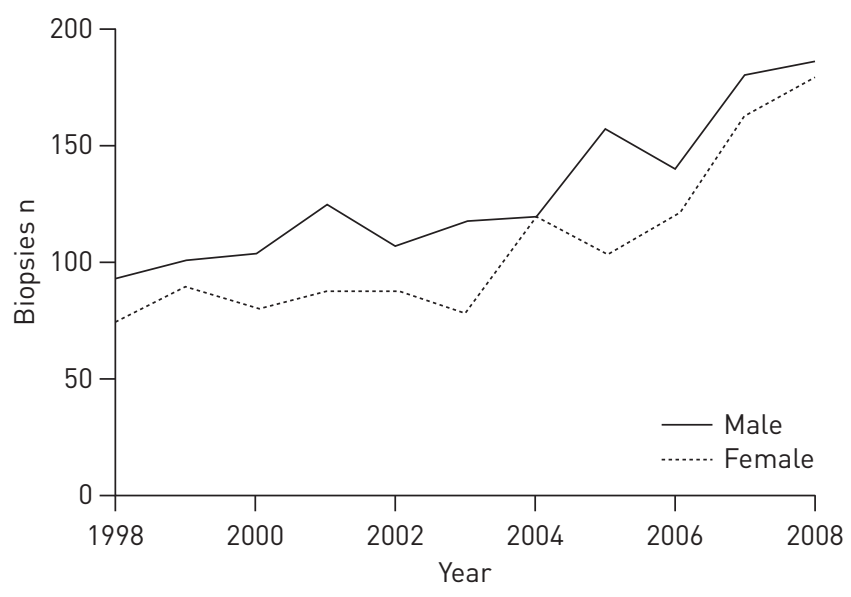




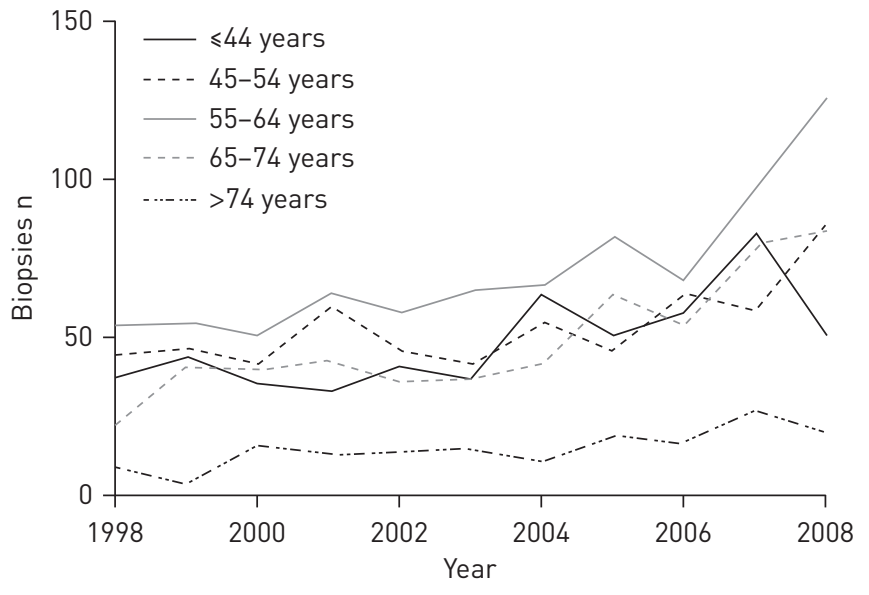

FIGURE 3 Number of biopsies over time, stratified by age category.

be interpreted with caution, as this originated from index admissions where final histology would likely not be available prior to discharge, and the confidence of this presumptive diagnosis may vary depending on the experience of the doctor completing discharge paperwork.

Our comorbidity assessment depended on the detail recorded in discharge records and may not have detected all medical problems. We did not have information on medications such as corticosteroids, immunosuppression, anticoagulation, and importantly pre-operative oxygen requirements and lung function, all of which have been associated with adverse outcomes in surgical lung biopsy case series $[6,19,20]$. Our mortality data only included the underlying cause of death, which may not reflect the "mode" of death, and therefore the majority of our cases with "ILD" may or may not have had pneumonia, acute exacerbations or sepsis as a final pathway. Finally, our data covered a time period lasting until 2008, and therefore it is likely that surgical practice and patient selection today would be slightly different.

Our estimates for mortality seem comparable to others in the literature. In fact, our overall in-hospital mortality of $1.7 \%$ is identical to that for elective patients from our recent large database study from the USA [10], where we identified similar risk factors for increased mortality. Although the distinction between whether biopsies were elective or nonelective was less robust in this study, our estimate for elective in-hospital mortality of $1.0 \%$ is slightly lower than in the US data, which may reflect a more cautious approach to biopsies in the UK. Our overall figure of $2.4 \% 30$-day mortality is similar to that reported by CARriLlo et al. [21] in the next largest series, of 722 patients in Mexico from 1986 to 1990, and also Sigurdsson et al. [20] in a smaller nationwide series from Iceland from 1986 to 2007. It is slightly higher than the $2.1 \%$ estimate for VATS procedures from a systematic review by NGUYEN and MEYER [7]

\section{TABLE 2 Primary diagnosis of readmissions within 3 months (for first readmission only)}

$\begin{array}{lc}\text { Total readmissions } & 397(100.0) \\ \text { Interstitial lung disease } & 202(50.9) \\ \text { Pneumonia/lower respiratory tract infection } & 38(9.6) \\ \text { Pneumothorax } & 19(4.8) \\ \text { Specified post-procedural issue } & 15(3.8) \\ \quad \text { Haemorrhage complicating a procedure } & 2(0.5) \\ \quad \text { Infection following a procedure } & 5(1.3) \\ \quad \text { Other complication of procedure } & 8(2.0) \\ \text { Cardiac problem le.g. myocardial infarction) } & 16(4.0) \\ \text { Other respiratory symptoms le.g. "cough") } & 10(2.5) \\ \text { Chest pain - not otherwise classified } & 8(2.0) \\ \text { Other infection le.g. urinary tract infections) } & 7(1.8) \\ \text { Pulmonary embolism } & 5(1.3) \\ \text { Pyothorax } & 2(0.5) \\ \text { Pleural effusion/haemothorax } & 4(1.0) \\ \text { Respiratory failure - other } & 3(0.8) \\ \text { Other respiratory - likely unrelated (e.g. CoPD) } & 8(2.0) \\ \text { Other - unrelated } & 60(15.1)\end{array}$

Data are presented as $n(\%)$. COPD: chronic obstructive pulmonary disease. 
TABLE 3 Cause of death of biopsy patients

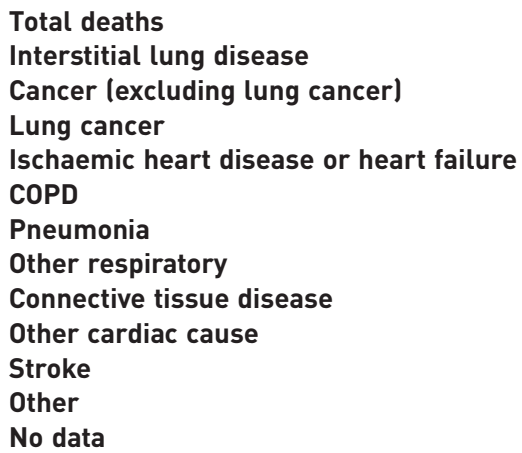

$911(100.0)$

$451(49.5)$

$94(10.3)$

$71(7.8)$

$53(5.8)$

$35(3.8)$

$35(3.8)$

$29(3.2)$

$25(2.7)$

$21(2.3)$

$13(1.4)$

$65(7.1)$

$19(2.1)$

Data are presented as $\mathrm{n}(\%)$. COPD: chronic obstructive pulmonary disease.

and the 1.5\% estimate from a recent case series in Edinburgh, UK [4], but lower than a previous summary estimate reported by KREIDER et al. [6] (4.5\%) (although this included studies with varying mortality end-points). Although comparison of mortality figures depends on case mix, end-points and procedure types, this consistency supports our estimates, and the comparison to mortality post-lobectomy is not insignificant [16]. 90-day mortality was less commonly reported in case series, but our overall estimate of $3.9 \%$ was again similar to that in Sigurdsson et al. [20].

TABLE 4 Multivariable analysis: associations with death within 90 days of biopsy

\begin{tabular}{|c|c|c|c|c|c|c|}
\hline & Cases & Deaths & Unadjusted OR $(95 \% \mathrm{CI})$ & p-value & Adjusted OR (95\% CI) & p-value \\
\hline \multicolumn{7}{|l|}{ Sex } \\
\hline Female & 1274 & 36 & 1.00 & 0.005 & 1.00 & \\
\hline \multicolumn{7}{|l|}{ Age group years } \\
\hline$\leqslant 44$ & 576 & 10 & 1.00 & \multirow[t]{3}{*}{$<0.001$ ( $\left.p_{\text {trend }}\right)$} & 1.00 & \multirow[t]{3}{*}{$<0.001\left(p_{\text {trend }}\right)$} \\
\hline $45-54$ & 636 & 21 & $1.93(0.90-4.14)$ & & $1.72(0.79-3.72)$ & \\
\hline$>74$ & 177 & 17 & $6.01(2.70-13.39)$ & & $4.33(1.90-9.89)$ & \\
\hline \multicolumn{7}{|l|}{ Updated Charlson score ${ }^{\#}$} \\
\hline 0 & 1947 & 75 & 1.00 & \multirow[t]{4}{*}{0.082 ( $\left.p_{\text {trend }}\right)$} & 1.00 & \multirow[t]{4}{*}{$0.037\left(p_{\text {trend }}\right)$} \\
\hline 1 & 717 & 23 & $0.83(0.51-1.33)$ & & $1.02(0.61-1.69)$ & \\
\hline 2 & 116 & 8 & $1.85(0.87-3.93)$ & & $1.63(0.75-3.54)$ & \\
\hline$\geqslant 3$ & 40 & $-^{+}$ & $3.57(1.36-9.36)$ & & $3.88(1.43-10.58)$ & \\
\hline \multicolumn{7}{|l|}{ Type of operation } \\
\hline \multicolumn{7}{|l|}{ Provisional diagnosis $f$} \\
\hline J84.1 & 1433 & 76 & 1.00 & \multirow[t]{7}{*}{$<0.001$} & 1.00 & \multirow[t]{7}{*}{0.008} \\
\hline J84.9 & 812 & 28 & $0.64(0.41-0.99)$ & & $0.76(0.48-1.20)$ & \\
\hline $\mathrm{J} 84.8$ & 99 & $-^{+}$ & $0.18(0.03-1.32)$ & & $0.23(0.03-1.65)$ & \\
\hline RA-ILD & 16 & 0 & & & & \\
\hline CTD-ILD & 38 & $-^{+}$ & $0.99(0.23-4.20)$ & & $0.91(0.21-3.94)$ & \\
\hline $\mathrm{HP}$ & 162 & $-^{+}$ & $0.34(0.11-1.08)$ & & $0.45(0.13-1.55)$ & \\
\hline Sarcoidosis & 260 & $-^{+}$ & $0.07(0.01-0.50)$ & & $0.10(0.01-0.74)$ & \\
\hline
\end{tabular}

Data are presented as n, unless otherwise stated. OR: odds ratio; IMD: Index of Multiple Deprivation 2010; VATS: video-assisted thoracoscopic surgery. " : higher updated Charlson score reflects greater degree of comorbidity; ": lower IMD score reflects least deprived; ${ }^{+}: 1-5$ (small numbers hidden to aid confidentiality); ${ }^{\S}$ : mainly reflects older data from before a specific code for VATS was available (these cases are likely

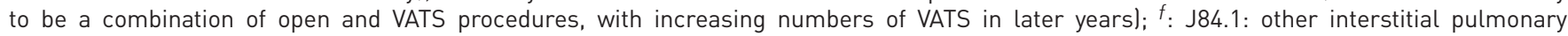
disease with fibrosis; J84.9: interstitial pulmonary disease, unspecified; J84.8: other specified interstitial pulmonary disease; RA-ILD ( J99.0): rheumatoid lung disease; CTD-ILD ( J99.1): respiratory disorders in other diffuse connective tissue disorders; HP (J67.9): hypersensitivity pneumonitis due to unspecified organic dust; sarcoidosis (D86.0): sarcoidosis of lung. 
Although our estimate of the proportion of cases of IPF undergoing lung biopsy may seem low, it is consistent with our experience that surgical biopsy is infrequently used to diagnose IPF in the UK if imaging is supportive. The proportion of younger patients undergoing biopsy also seems higher than would be expected; however, again this is consistent with our experience that biopsy will be more readily attempted in younger patients with low comorbidity, whereas clinicians may be more reluctant to offer the procedure to older patients. It is possible though that our caseload may include more younger patients with an inflammatory type of ILD. Given we excluded patients with lung cancer, it is surprising that so many patients eventually died of this: this may reflect uncoded disease before the biopsy, but also that lung cancer is more common in patients with IPF and may develop later on. As noted, if we omitted all patients who ultimately died from any type of cancer, overall mortality was essentially no different (in-hospital, 30-day and 90-day mortality of 1.7\%, 2.4\% and 4.0\%, respectively). Given our data includes patients who underwent open surgery (which has a higher mortality) it is likely that our figures will slightly overestimate the expected mortality from a VATS procedure carried out today.

The increasing number of biopsies over time in our study may suggest an increasing desire to characterise ILD, but may simply reflect the rising incidence of ILD in the UK $[15,22]$. It would be useful to extend our analysis to the present day to assess the impact of the availability of targeted treatments for IPF on rates of biopsy.

The mortality following surgical biopsy in ILD, combined with improvements and greater experience in imaging studies, suggests that the decision to undergo biopsy should be taken very carefully in older patients with comorbidities, with clear counselling of risks and awareness of factors associated with poorer outcomes. The limited number of biopsies performed annually per surgical centre in England suggests a national audit would be an effective means of monitoring outcomes: the Society for Cardiothoracic Surgeons now collates some of this data for UK centres, and this could be used to monitor local practice. The possible impact of low surgical centre volume on mortality may be less relevant for thoracic surgery in the UK [23], but should be taken into consideration in other regions [24].

In conclusion, our data suggests there were increasing numbers of surgical lung biopsies for ILD in England from 1997 to 2008, with 4-5\% of new cases of IPF-clinical syndrome being biopsied. 30-day and 90 -day mortality were estimated at $2.4 \%$ and $3.9 \%$, respectively. Increasing age and comorbidity were risk factors for adverse outcome. Our data suggests a patient aged $<65$ years with no significant comorbidities has a 30 -day mortality of $1.6 \%$, whereas a patient aged $\geqslant 65$ years with comorbidities has a 30 -day mortality of $4.7 \%$. Clearly personal factors such as lung function tests need to be taken into account, but these risks need to be discussed with patients.

\section{Acknowledgements}

Authors contributions are as follows: T.M. McKeever, A.W. Fogarty, V. Navaratnam and R.B. Hubbard conceived the study; V. Navaratnam and J.P. Hutchinson requested and had access to the data; J.P. Hutchinson prepared and analysed the data, and wrote the first draft. All authors were involved in reviewing the study and developing the final draft. All approved the final draft prior to submission.

\section{References}

1 Travis WD, Costabel U, Hansell DM, et al. An official American Thoracic Society/European Respiratory Society statement: update of the international multidisciplinary classification of the idiopathic interstitial pneumonias. Am J Respir Crit Care Med 2013; 188: 733-748.

2 Raghu G, Collard HR, Egan JJ, et al. An official ATS/ERS/JRS/ALAT statement: Idiopathic pulmonary fibrosis: evidence-based guidelines for diagnosis and management. Am J Respir Crit Care Med 2011; 183: 788-824.

3 Raghu G, Rochwerg B, Zhang Y, et al. An official ATS/ERS/JRS/ALAT clinical practice guideline: treatment of idiopathic pulmonary fibrosis. An update of the 2011 clinical practice guideline. Am J Respir Crit Care Med 2015; 192: e3-e19.

4 Morris D, Zamvar V. The efficacy of video-assisted thoracoscopic surgery lung biopsies in patients with interstitial lung disease: a retrospective study of 66 patients. J Cardiothorac Surg 2014; 9: 45.

5 Blackhall V, Asif M, Renieri A, et al. The role of surgical lung biopsy in the management of interstitial lung disease: experience from a single institution in the UK. Interact Cardiovasc Thorac Surg 2013; 17: 253-257.

6 Kreider ME, Hansen-Flaschen J, Ahmad NN, et al. Complications of video-assisted thoracoscopic lung biopsy in patients with interstitial lung disease. Ann Thorac Surg 2007; 83: 1140-1144.

7 Nguyen W, Meyer KC. Surgical lung biopsy for the diagnosis of interstitial lung disease: a review of the literature and recommendations for optimizing safety and efficacy. Sarcoidosis Vasc Diffuse Lung Dis 2013; 30: 3-16.

8 Utz JP, Ryu JH, Douglas WW, et al. High short-term mortality following lung biopsy for usual interstitial pneumonia. Eur Respir J 2001; 17: 175-179.

9 Lee YC, Wu CT, Hsu HH, et al. Surgical lung biopsy for diffuse pulmonary disease: experience of 196 patients. J Thorac Cardiovasc Surg 2005; 129: 984-990.

10 Hutchinson JP, Fogarty AW, McKeever TM, et al. In-hospital mortality following surgical lung biopsy for interstitial lung disease in the USA: 2000-2011. Am J Respir Crit Care Med 2016; 193: 1161-1167.

11 Health and Social Care Information Centre. Hospital Episodes Statistics. www.hscic.gov.uk/hes Date last accessed: October 31, 2015.

12 Quan H, Li B, Couris CM, et al. Updating and validating the Charlson comorbidity index and score for risk adjustment in hospital discharge abstracts using data from 6 countries. Am J Epidemiol 2011; 173: 676-682. 
13 Charlson ME, Pompei P, Ales KL, et al. A new method of classifying prognostic comorbidity in longitudinal studies: development and validation. J Chronic Dis 1987; 40: 373-383.

14 UK Department for Communities and Local Government. English Indices of Deprivation 2010. www.gov.uk/ government/statistics/english-indices-of-deprivation-2010 Date last accessed: September 30, 2014.

15 Navaratnam V, Fleming KM, West J, et al. The rising incidence of idiopathic pulmonary fibrosis in the U.K. Thorax 2011; 66: 462-467.

16 Powell HA, Tata LJ, Baldwin DR, et al. Early mortality after surgical resection for lung cancer: an analysis of the English national lung cancer audit. Thorax 2013; 68: 826-834.

17 Blanco M, Obeso GA, Duran JC, et al. Surgical lung biopsy for diffuse lung disease. Our experience in the last 15 years. Rev Port Pneumol 2013; 19: 59-64.

18 American Thoracic Society and European Respiratory Society. American Thoracic Society/European Respiratory Society international multidisciplinary consensus classification of the idiopathic interstitial pneumonias. Am $J$ Respir Crit Care Med 2002; 165: 277-304.

19 Mouroux J, Clary-Meinesz C, Padovani B, et al. Efficacy and safety of videothoracoscopic lung biopsy in the diagnosis of interstitial lung disease. Eur J Cardiothorac Surg 1997; 11: 22-26,

20 Sigurdsson MI, Isaksson HJ, Gudmundsson G, et al. Diagnostic surgical lung biopsies for suspected interstitial lung diseases: a retrospective study. Ann Thorac Surg 2009; 88: 227-232.

21 Carrillo G, Estrada A, Pedroza J, et al. Preoperative risk factors associated with mortality in lung biopsy patients with interstitial lung disease. J Invest Surg 2005; 18: 39-45.

22 Hutchinson JP, McKeever TM, Fogarty AW, et al. Increasing global mortality from idiopathic pulmonary fibrosis in the twenty-first century. Ann Am Thorac Soc 2014; 11: 1176-1185.

23 Treasure T, Utley M, Bailey A. Assessment of whether in-hospital mortality for lobectomy is a useful standard for the quality of lung cancer surgery: retrospective study. BMJ 2003; 327: 73.

24 Birkmeyer JD, Siewers AE, Finlayson EV, et al. Hospital volume and surgical mortality in the united states. $N$ Engl J Med 2002; 346: 1128-1137. 